\title{
The Drosophila nephrocyte has a glomerular filtration system
}

Fujian Zhang and Xiangmei Chen

We read with interest the Review by Paola Romagnani and colleagues (Renal progenitors: an evolutionary conserved strategy for kidney regeneration. Nat Rev Nephrol. 9, 137-146; 2013), ${ }^{1}$ which discusses the importance of the evolutionarily conserved nephron structure in kidney regeneration. Nephrology students who read this paper will get the incorrect impression that insects have renal tubular structures but do not have a glomerular filtration system. This oversight is reasonable because the filtration system has only recently been discovered in Drosophila melanogaster and its existence has not yet been fully accepted in nephrology research. ${ }^{2,3}$

The nephron is the basic functional and structural unit of the kidney; the glomerulus controls ultrafiltration and the renal tubule is responsible for reabsorption and secretion. ${ }^{4,5}$ The Drosophila excretory system is composed of Malpighian tubules and nephrocytes. ${ }^{6}$ The Malpighian tubules are responsible for urine modification and secretion, and have been extensively studied in the past two decades. ${ }^{7.8}$ However, the first evidence that insects have podocytelike cells was obtained fairly recently. In 2009, Weavers et al. showed that the Drosophila nephrocyte has a highly specialized filtration structure-the nephrocyte diaphragm - with remarkable similarities to the slit diaphragm of mammalian glomerular podocytes. ${ }^{2}$ In the Drosophila nephrocyte, foot processes created by infolding of the plasma membrane generate $30 \mathrm{~nm}$ slit pores that are comparable to those created by podocyte foot processes in the mammalian glomerulus. The nephrocyte diaphragms, together with a basement membrane that covers the entire nephrocyte, form a size-selective and charge-selective filtration barrier that filters haemolymph. In Drosophila, two different types of nephrocyte are present: pericardial nephrocytes flank the heart tube and garland nephrocytes wrap around the oesophagus. At the embryonic stage the garland nephrocytes are the only functional filtration cells; pericardial nephrocytes are the primary filtration cells at the larval and adult stages. ${ }^{2,3}$

Our research group has developed a reliable functional readout for Drosophila nephrocytes in vivo and established an RNA interference system to identify genes that are required for nephrocyte function. ${ }^{9}$ Using this system, we performed large-scale screens and showed that Drosophila nephrocytes share remarkable functional, structural and molecular similarities with mammalian renal proximal tubule cells. ${ }^{10}$ Both Drosophila nephrocytes and renal proximal tubule cells have high endocytosis activity, and nephrocytes use orthologues of mammalian cubilin and amnionless-the two major receptors for protein reabsorption in the renal proximal tubule - to take up proteins from haemolymph. Similar to renal proximal tubules, numerous endocyticrelated organelles, such as endosomes, lysosomes and large vacuoles, are present in Drosophila nephrocytes. The lacuna structures of the nephrocytes resemble the brush border structures of renal proximal tubules, which provide a large membrane surface for protein reabsorption. Taken together, these findings suggest that the Drosophila nephrocyte not only has glomerular filtration function but is able to reabsorb protein in a similar way to the renal proximal tubule.

The availability of powerful genetic tools and the tremendous genetic resources of Drosophila make it an ideal model for largescale screens to identify and study genes that are involved in normal physiological functions or diseases of various organs. Our understanding of the genetic network required for renal function has been hindered by the relative intractability of characteristics of mammalian nephron structure and function in vivo. With the emergence of Drosophila nephrocytes as an ideal model system to study renal biology, we expect that novel genes will be identified with unprecedented speed and studied in great detail, facilitating our understanding of genetic kidney diseases.

To date, whether mammalian glomerular podocytes and renal proximal tubular cells are derived from the same progenitor cells during embryonic kidney development remains unknown. ${ }^{1}$ The ability of a murine kidney-derived stem cell line to differentiate into either podocytes or renal proximal tubule cells has been reported, suggesting that these cells are developmentally related. ${ }^{11}$ The fact that the Drosophila nephrocyte shares remarkable similarities with both mammalian glomerular podocytes and renal proximal tubules suggests that these cell types have the same evolutionary origin. Whether nephrocyte-like progenitor cells exist in Drosophila, and whether such cells could perform a role in the regeneration of nephrocytes after injury, is not clear. ${ }^{12,13}$ In the future, it would be very interesting to examine whether a cellular regeneration or nephron neogenesis process occurs in Drosophila nephrocytes after injury, and explore whether nephrocyte-like progenitor cells exist in this organism.

Department of Nephrology, Chinese PLA General Hospital, State Key Laboratory of Kidney Diseases, National Clinical Research Center of Kidney Diseases, 28 Fuxing Road, Beijing 100853, China (F.Z., X.C.).

Correspondence to:

F.Z.

fujianzhang@yahoo.com

X.C.

xmchen301@126.com

Acknowledgements

X.C.'s work is supported by the National

Basic Research Program of China (973 Program, No. 2013CB530800).

Competing interests

The authors declare no competing interests.

1. Romagnani, P., Lasagni, L. \& Remuzzi, G. Renal progenitors: an evolutionary conserved strategy for kidney regeneration. Nat. Rev. Nephrol. 9, 137-146 (2013).

2. Weavers, H. et al. The insect nephrocyte is a podocyte-like cell with a filtration slit diaphragm. Nature 457, 322-326 (2009). 


\section{CORRESPONDENCE}

3. Zhuang, S. et al. Sns and Kirre, the Drosophila orthologs of Nephrin and Neph1, direct adhesion, fusion and formation of a slit diaphragm-like structure in insect nephrocytes. Development 136, 2335-2344 (2009).

4. Gubler, M. C. Nephrotic syndrome: genetic testing in steroid-resistant nephrotic syndrome. Nat. Rev. Nephrol. 7, 430-431 (2011).

5. Hildebrandt, F. Genetic kidney diseases. Lancet 375, 1287-1295 (2010)

6. Cagan, R. L. The Drosophila nephrocyte. Current Opin. Nephrol. Hypertens. 20, 409-415 (2011).
7. Dow, J. A. \& Romero, M. F. Drosophila provides rapid modeling of renal development, function, and disease. Am. J. Physiol. Renal Physiol. 299 F1237-F1244 (2010).

8. Jung, A. C., Denholm, B., Skaer, H. \& Affolter, M. Renal tubule development in Drosophila: a closer look at the cellular level. J. Am. Soc. Nephrol. 16, 322-328 (2005).

9. Zhang, F., Zhao, Y. \& Han, Z. An in vivo functional analysis system for renal gene discovery in Drosophila pericardial nephrocytes. J. Am. Soc. Nephrol. 24, 191-197 (2013).

10. Zhang, F., Zhao, Y., Chao, Y., Muir, K. \& Han, Z. Cubilin and amnionless mediate protein reabsorption in Drosophila nephrocytes. J. Am. Soc. Nephrol. 24, 209-216 (2013).

11. Fuente Mora, C. et al. Differentiation of podocyte and proximal tubule-like cells from a mouse kidney-derived stem cell line. Stem Cells Dev. 21, 296-307 (2012).

12. Singh, S. R. \& Hou, S. X. Multipotent stem cells in the Malpighian tubules of adult Drosophila melanogaster. J. Exp. Biol. 212, 413-423 (2009).

13. Singh, S. R. \& Hou, S. X. Lessons learned about adult kidney stem cells from the malpighian tubules of Drosophila. J. Am. Soc. Nephrol. 19, 660-666 (2008). 